Revue des patrimoines

25 | 2014

Le patrimoine de la Grande Guerre

\title{
D'un tombeau vide à une tribune politique : genèse et évolution d'un espace commémoratif majeur
}

Jérémy Pignard

\section{(2) OpenEdition}

1 Journals

Electronic version

URL: http://journals.openedition.org/insitu/11386

DOI: 10.4000/insitu.11386

ISSN: 1630-7305

Publisher

Ministère de la Culture

\section{Electronic reference}

Jérémy Pignard, «D'un tombeau vide à une tribune politique : genèse et évolution d'un espace commémoratif majeur », In Situ [Online], 25 | 2014, Online since 09 December 2014, connection on 25 June 2020. URL : http://journals.openedition.org/insitu/11386; DOI : https://doi.org/10.4000/insitu. 11386

This text was automatically generated on 25 June 2020 .

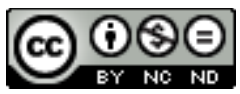

In Situ Revues des patrimoines est mis à disposition selon les termes de la licence Creative Commons Attribution - Pas d'Utilisation Commerciale - Pas de Modification 4.0 International. 


\title{
D'un tombeau vide à une tribune politique : genèse et évolution d'un espace commémoratif majeur
}

\author{
Jérémy Pignard
}

1 En France, la quasi-totalité des communes a un monument aux morts qui s'élève sur la place principale, au cimetière ou devant l'église. Édifiés à la demande des mairies, des paroisses et sous la pression de la population, ces constructions ont servi d'expiatoire au deuil submergeant la nation au lendemain de la Première Guerre mondiale. Mais ces constructions ont aussi permis aux familles d'avoir une tombe symbolique se substituant à la véritable sépulture, celle qui détient le corps (pour les soldats qui ont pu être identifiés). L'ampleur de ce conflit et l'intensité des combats ont marqué les esprits. On compte 1,2 million de morts, des millions de blessés, d'amputés, de gueules cassées, des villes et des villages anéantis par les bombardements et presque toute la société française est touchée par le deuil. À cela s'ajoute un impact psychologique et moral très lourd. Le retour à un monde en paix en 1918-1919 n'est en aucun cas un retour à la vie d'avant-guerre car il n'est plus de comparaison possible avec la «Belle Époque ». C'est dans ce contexte qu'apparaît le monument aux morts qui permet dès lors d'exorciser ce changement brutal de la société française. Il rassure d'une part les endeuillés en leur promettant de garder intact le nom de leurs disparus, et d'autre part il rassemble la société d'après-guerre.

2 C'est la principale raison d'être de ces monuments. Mais il serait trop simple de penser qu'il ne s'agit que d'une forme de «copier-coller» que l'on pourrait retrouver dans chaque commune de France. En réalité chaque monument est une œuvre unique, ne serait-ce que par les noms qu'il porte. Chaque édifice devient ainsi une part d'un patrimoine communal que les générations suivantes se sont attachées à faire perdurer. Collectivement ils sont un témoignage de l'histoire de France, un patrimoine national qui cherche à expliquer ce que fut la guerre, ses horreurs et ses conséquences. De fait ces constructions revêtent deux niveaux de patrimonialité qui expliquent en partie l'importance toujours très grande qui leur est accordée aujourd'hui. De plus, dès sa 
création le monument aux morts s'identifie à une tribune politique permettant à des élus locaux d'exprimer clairement leurs prises de position tout en relayant des idées des gouvernants nationaux. Enfin, ces édifices sont demeurés des espaces symboliques forts car ils ont su réaliser ce que nous pourrions appeler un « syncrétisme mémoriel » en intégrant à la mémoire des soldats de la Grande Guerre celles de la Seconde Guerre mondiale, de la guerre d'Algérie et désormais celle des soldats professionnels tombés au cours des opérations sur des théâtres extérieurs (Afghanistan, Mali...).

3 Pour tenter d'expliciter au mieux ces éléments, cette courte analyse s'appuie essentiellement sur des monuments situés dans le département de l'Isère. Toutefois, chaque monument, chaque symbole ou chaque idée peut se retrouver dans nombre d'autres édifices présents sur le territoire français. Objet très riche, les monuments aux morts peuvent être analysés sous de nombreux angles comme l'ont montré les historiens qui les ont étudiés. Antoine Prost en a notamment proposé une typologie précise $^{1}$. Nous ne verrons cependant pas ici toutes les facettes de ces édifices, à la fois simples dans leur lecture et complexes dans leur étude.

\section{De la plume au burin, la genèse du monument}

4 Aussitôt signée, l'annonce de l'armistice s'est diffusée dans le pays comme un soulagement, laissant éclater parfois une réelle euphorie et un esprit de fête. Pourtant ces instants de joie et de gaieté ne durent pas et la victoire apparaît rapidement comme amère, ce que met d'ailleurs très bien en évidence Bruno Cabanes dans sa thèse ${ }^{2}$ en montrant que le deuil est omniprésent, empêchant toute célébration de la victoire trop exacerbée. Il faut toutefois justifier le sang versé et rappeler les raisons de la guerre. La mort de ces nouveaux "héros" de la nation devient progressivement un exemple, notamment pour la République encore à la recherche de symboles pour ancrer sa présence dans le territoire. Dès lors une obligation morale se fait jour pour les survivants : ne pas oublier le sacrifice de ces centaines de milliers de personnes pour qu'il ne soit pas vain. Dans cette optique, émergent de nombreux élans de compassion envers les endeuillés et de respect presque admiratif pour les victimes. L'un des journaux locaux de la ville de Voiron en Isère se fait le relais de ces témoignages qui oscillent entre la blessure du deuil et la gloire patriotique du sacrifice :

Avant d'ouvrir le livre de douleur et de gloire qui tremble dans nos mains, avant d'évoquer le doux et fier souvenir de nos grands morts de la guerre, d'en faire l'appel suprême et d'apporter à chacun l'hommage profond de notre admiration et de notre reconnaissance, nous nous découvrons avec respect devant ceux qui les pleurent ${ }^{3}$.

5 Cet extrait montre bien cette dualité des sentiments à l'origine de la construction des monuments aux morts. Ces derniers doivent répondre clairement à une forme d'injonction: honorer ces " héros" tout en permettant à leurs proches de faire leur deuil. C'est ce que Stéphane Audouin-Rouzeau et Annette Becker ont bien montré, expliquant que ces monuments, en exaltant l'héroïsme, devaient aider les veuves, les orphelins et les anciens combattants à faire le travail de deuil après la guerre ${ }^{4}$. Ainsi dans les années 1920 sont érigés des édifices. Ils sont destinés à simplifier le deuil souvent rendu difficile voire impossible par l'absence du corps. Celui-ci était laissé dans les cimetières près du front lorsqu'une identification était possible. Il devenait ainsi 
une tombe de substitution et de fait un monument aux morts et pas un monument de la victoire, comme le rappelle Reinhart Koselleck dans L'Expérience de l'histoire ${ }^{5}$.

Pour répondre aux attentes de cet après-guerre, les projets ne manquent pas. Certains n'ont jamais vu le jour comme dans la commune de Moirans (Isère) où une pétition circule dès mars 1919 pour demander que l'église

reçoive les restes de ces héros ou que des plaques y soient placées pour honorer leur mémoire [...]. Dormant sous les dalles funéraires de la vieille église, plus près des yeux, ils seraient aussi plus près du cœur, et pourraient recevoir plus souvent les hommages privés et publics de notre pieux souvenir'.

7 Pourtant le projet n'est pas retenu du fait de la difficulté à rapatrier les corps mais aussi à cause de l'inégalité que cela engendrerait entre ceux dont le corps fut reconnu et les autres jamais identifiés.

8 La grande majorité des constructions revêtent avant tout un caractère funéraire. Leur but premier étant de remplacer une tombe individuelle de tous les soldats « morts pour la France ", ils sont rapidement imprégnés du caractère mortuaire. Celui-ci est très souvent mis en valeur au moment de l'inauguration du monument aux morts. Ainsi en est-il de celle du monument paroissial de Miribel-les-Échelles, en Isère. Le protocole utilisé a été calqué sur celui d'un enterrement comme en témoigne La République de l'Isère :

La décoration faite de tentures noires, de faisceaux de drapeaux tricolores et de guirlandes de buis était d'un goût parfait. Au milieu de la grande nef est dressée une tombe, image de la tombe du front, garnie de mousse avec une guirlande de fine bruyère entourant la croix, au sommet de laquelle est placé un casque de poilu? ${ }^{7}$.

Dans ce cas précis, la mise en scène sert à recréer une atmosphère de funérailles, comme pour donner à chaque soldat de la commune l'enterrement auquel il a droit. Les cérémonies sont souvent accompagnées de l'appel des morts ou encore d'une minute de silence, une forme laïcisée de prière selon Antoine Prost ${ }^{8}$. De plus le protocole cherche également à faire le lien entre le front et l'arrière en réutilisant l'image des tombes à proximité des champs de bataille, symbolisées par les croix de bois surmontées d'un képi ou d'un casque. De ce fait l'arrière rend hommage à ses morts à travers cette cérémonie et tout est fait pour rappeler la mort, le deuil avec l'édifice en lui-même. S'il est placé au cœur du cimetière, comme celui du Grand Lemps en Isère, le caractère funéraire en est de facto renforcé. Cela se retrouve également avec la symbolique sur les monuments comme celui de la commune d'Apprieu (fig. 1) surmonté d'une urne funéraire, ou encore la colonne brisée de Saint-Bueil (fig. 2) s'identifiant aux vies brisées par la guerre. Enfin certaines constructions sont encore bien plus explicites de cette symbolique : le plus emblématique d'entre eux est celui de la paroisse de Bévenais (fig. 3) décrit par La République de l'Isère en septembre 1919 :

C'est une peinture sur toile de 3 mètres sur 2 mètres 10 représentant un cimetière sur le front et un paysage de la Marne. Au milieu des tombes surmontées de la modeste croix de bois avec la cocarde tricolore; trois personnages d'un dessin et d'une expression remarquables frappent surtout : la France au regard à la fois triste et fier remet l'épée au fourreau et regarde avec confiance l'avenir ; une femme en deuil prie et pleure agenouillée sur la tombe de son enfant, et une fillette, le bras gauche chargé de fleurs, parcourt les tombes une à une pour les fleurir. À droite, en arrière, un village en flammes, et à gauche quelques maisons percées d'obus 9 . 
Figure 1

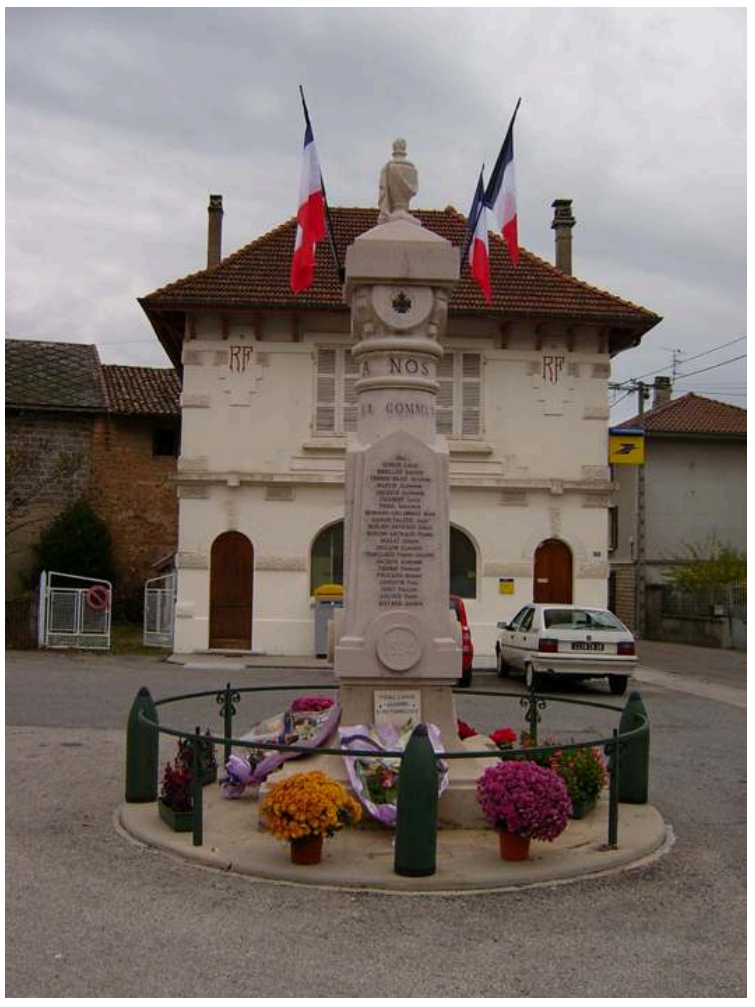

Apprieu (Isère), monument aux morts, 2006.

PHOT. PIgNARD, JÉRÉMY. @ JÉRÉMY PIgNARD. 
Figure 2

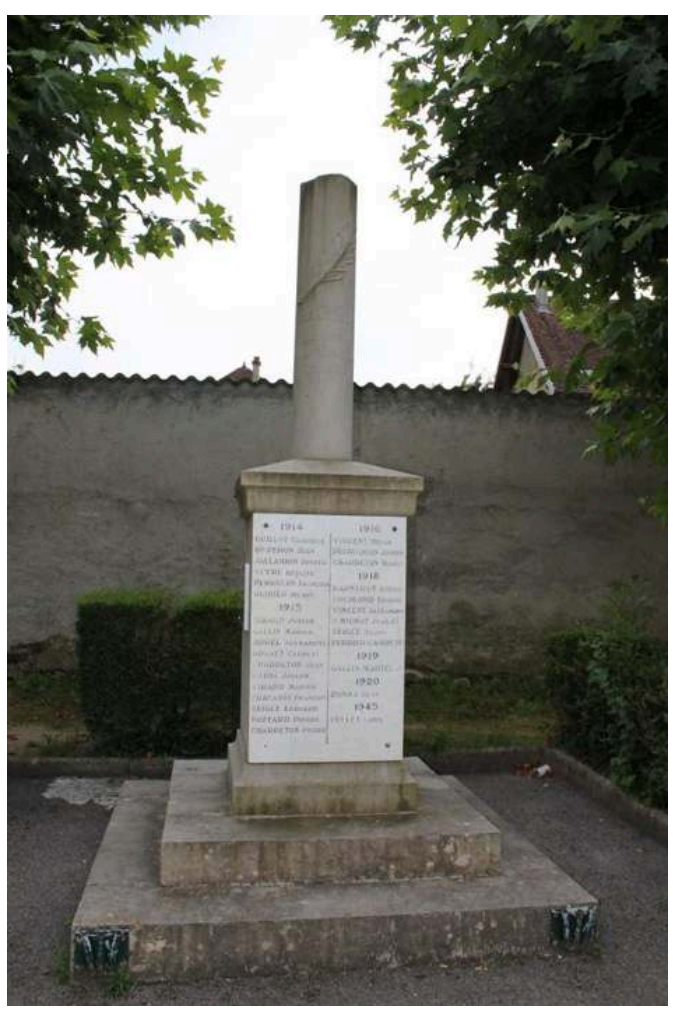

Saint-Bueil (Isère), monument aux morts, 2013.

PHOT. PIgNARD, JÉRÉMY. @ JéRÉMY PIgNARD.

Figure 3

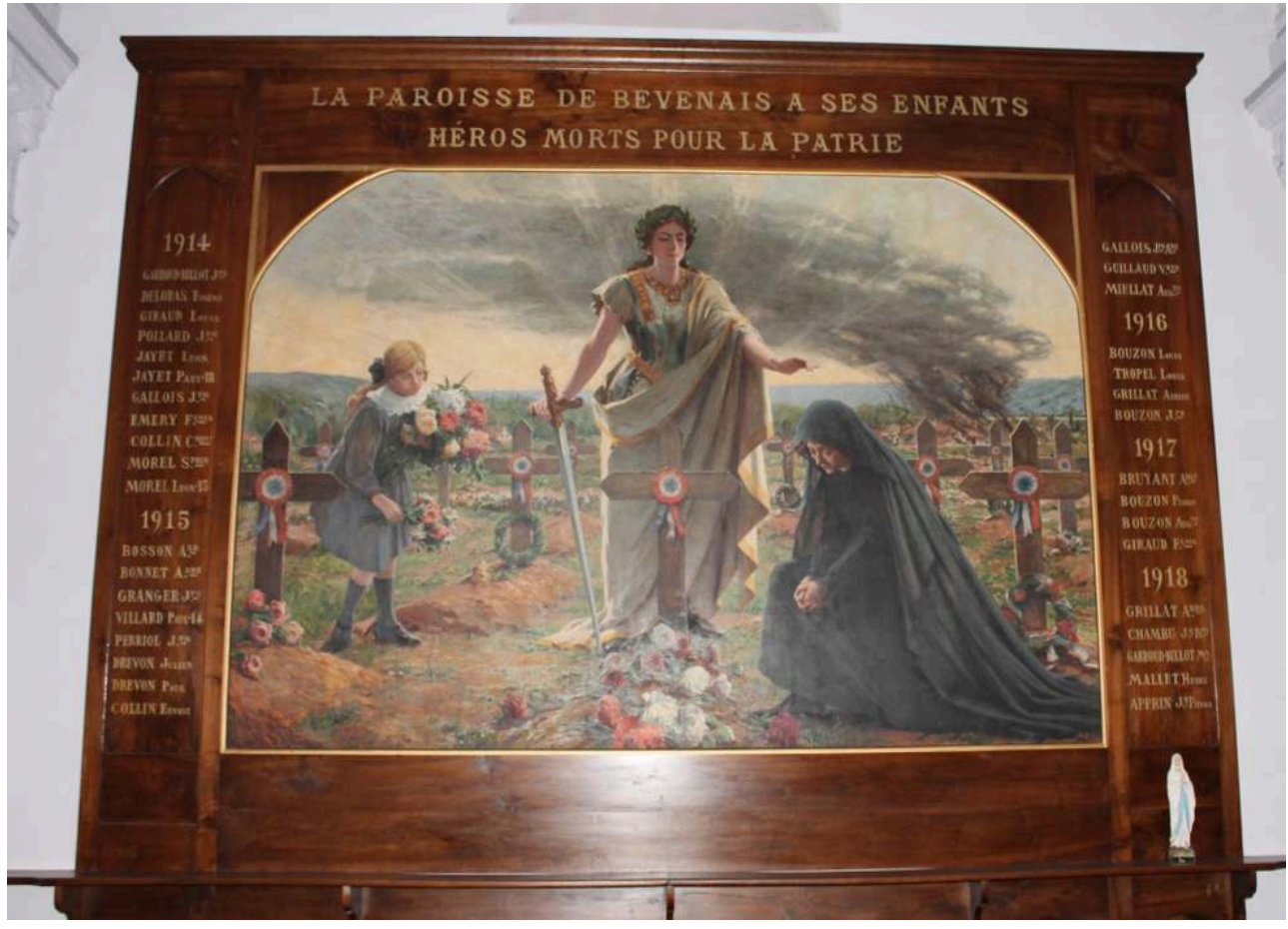

Paroisse de Bévenais (Isère), monument aux morts, 2013.

Phot. Pignard, Jérémy. (c) Jérémy Pignard. 

après-guerre; rien ne peut se faire sans référence à celui-ci. Toutefois ces édifices insistent sur d'autres caractéristiques du conflit ainsi que sur son impact sur la société d'après-guerre.

11 Comme cela a été dit précédemment, chacune de ces constructions a permis une double patrimonialité qui se retrouve à travers son utilisation et les discours tenus lors des commémorations, mais également et surtout avec l'épigraphie. L'analyse des inscriptions présentes sur ces édifices permet d'identifier ce caractère dual entre l'échelle nationale et l'échelle communale. Sur presque tous les monuments il est possible de lire la mention "mort pour la France "; celle-ci, définie par la loi du 2 juillet $1915^{10}$, met en évidence le lien avec le pays. En honorant des hommes et des femmes qui ont choisi de sacrifier leur vie pour la patrie, l'édifice est un élément du patrimoine national. En même temps ces mêmes édifices insistent sur une dimension communale qui se retrouve à travers la mention explicite à la ville ou au village qui essaient de «s'approprier» les morts à travers des inscriptions récurrentes et formalisées. Ainsi en est-il des formules « aux enfants de » suivi du nom de la commune ou encore «à ses morts». Ces inscriptions font du monument un élément du patrimoine communal dans le sens où le mort, et désormais sa mémoire, appartiennent à la commune. Pour ce faire, il devient indispensable de préciser de quels morts il faut garder la mémoire. personnes dont il entretient la mémoire. Cela permet d'individualiser le deuil et l'hommage alors même que le monument exprime une démarche et une commémoration collectives. Ceux qui ont élaboré ces constructions ont connu ces morts et pour les anciens combattants, ont partagé des expériences de combats. Il y a une forme d'obligation collective à se souvenir à la fois des défunts et des circonstances dans lesquelles ils ont trouvé la mort. Ainsi le maire de Lux en Côte-d'Or, dans son discours du 11 novembre 1926, insiste sur ce lien qui existe entre les vivants et les morts :

En ce jour de victoire, le culte de nos glorieux morts est un devoir sacré. Parmi ces morts il en est vers qui notre pensée doit se porter tout particulièrement. Ceux dont nous honorons aujourd'hui la mémoire : vos pères, vos fils, vos époux, vos frères, nos compagnons de jeunesse, nos amis qui, partis pleins d'entrain, pour la défense de la cause sacrée, sont tombés à jamais loin du cher pays natal ${ }^{11}$.

13 Pourtant ce sentiment s'estompe ou se modifie avec les générations successives. Cellesci s'attachent davantage à commémorer les horreurs de la guerre qu'à se souvenir de personnes qu'ils n'ont jamais connues; la lecture des noms devient dès lors un autre moyen d'insister sur les conséquences néfastes des guerres. Ces listes, presque toujours organisées par ordre alphabétique ou chronologique, ne comportent généralement que ceux qui sont morts au combat et rares sont les monuments qui célèbrent aussi les anciens combattants. Dans la commune de Charancieu en Isère, il a pourtant été fait le choix de commémorer ceux qui ont combattu et qui ont survécu à la guerre (fig. 4) ; leur nom fut ajouté sur l'édifice au moment de leur décès, parfois bien des décennies après la guerre. Cela transforme nécessairement l'usage de l'édifice. Celui-ci cesse d'être un objet mémoriel de ceux qui sont morts au combat et devient davantage un édifice célébrant l'engagement de la commune dans un conflit national. 
Figure 4

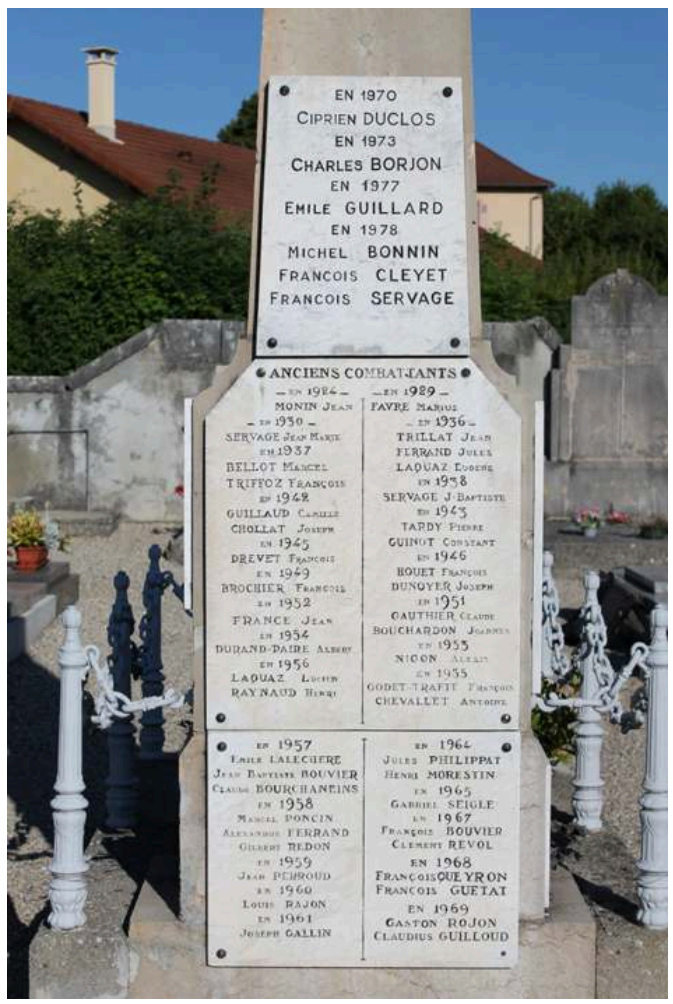

Charancieu (Isère), détail du monument aux morts, 2013.

Phot. Pignard, Jérémy. (c) Jérémy Pignard.

14 Ainsi le monument aux morts s'est imposé dès l'immédiat d'après-guerre comme l'espace commémoratif par excellence et les contestations ont été faibles. Pour autant toute la société n'adhère pas toujours unanimement à ces projets de construction. Dans certains cas, c'est le choix du monument qui est contesté comme à Voiron en Isère. L'édifice a été choisi par un jury, désigné par le Comité du monument aux morts, auquel il a été proposé plusieurs projets ; or si le choix s'est porté sur la maquette intitulée «Patrie » de Gaston Dintrat, le Petit Voironnais rapporte que celle-ci «n'a pas été du goût de la population voironnaise ${ }^{12} »$. Les édifices ne font pas toujours l'unanimité du fait des affinités politiques, des croyances religieuses ou encore des sensibilités artistiques. Les catholiques souhaitent la présence de symboles religieux, ce qui est interdit en raison de la loi récente de séparation des Églises et de l'État. Les personnes de sensibilité radicale ou socialiste ont tendance à refouler les édifices qui figure un poilu triomphant, un symbole trop cocardier selon eux. Parfois ces désaccords se transforment en véritable opposition qui peut aller jusqu'à une bagarre. L'un des exemples les plus emblématiques de ce cas de figure est celui de l'inauguration du monument aux morts de la commune de Saint-Aupre en Isère. Alors que l'inauguration arrivait à son terme, une contestation se fit entendre comme l'explique le Petit Voironnais du 2 septembre 1923 :

Voulant renouveler sa tentative si piteusement échouée à Coublevie [commune voisine], la même bande d'énergumènes communistes qui a rêvé de troubler toutes les fêtes semblables de commémoration aux morts de la guerre, après le cri de «vive l'Internationale» [...] voulut escalader l'estrade et organiser une manifestation antipatriotique. Mais elle avait compté sans le sentiment généreux des habitants de Saint-Aupre. Ceux-ci, avisés de la venue de cette troupe de voyous 
anarchistes, ne permirent pas qu'ils pussent esquisser même un seul geste. En un clin d'œil ils leur distribuèrent la plus formidable correction qu'on puisse imaginer : gifles, coup de poing, bottes au derrière, ces individus durent encaisser simultanément tout cela avant de fuir de toute la vitesse de leurs jambes, et même au cours de cette fuite, car ils ne furent pas lâchés par leurs justiciers ${ }^{13}$.

Le monument aux morts ne plaît pas à toutes les parties de la société et certains opposants se montrent virulents. Malgré cela, l'édifice progressivement convainc. Loin d'être une construction à la gloire des actions militaires, il devient le pôle de la mémoire collective des victimes de la guerre et de la défense du pacifisme. Le monument n'étant pas immuable, il revêt des caractéristiques différentes en fonction du contexte. L'édifice en lui-même transmet des idées mais son véritable message vient de la manière dont il est utilisé.

\section{De la commémoration au « syncrétisme mémoriel »}

Face au deuil au lendemain de la Grande Guerre, le monument aux morts seul ne suffit pas. Il est nécessaire d'organiser la commémoration des victimes de ce conflit afin de mieux l'encadrer. L'État a déjà pris certaines dispositions comme l'a bien montré Serge Barcellini ${ }^{14}$. Ce dernier montre que la première est la loi du 2 juillet 1915 attribuant la mention «mort pour la France». S'ensuivent plusieurs autres lois. Celle du 25 novembre 1918 crée l'Office des sépultures militaires. Celle du 19 octobre 1919 sur la " commémoration et la glorification des morts pour la France au cours de la Grande Guerre », se caractérise par la création des livres d'or et la mise en place de subventions pour les monuments aux morts. Enfin, le décret du 17 janvier 1920 institue le ministère des Pensions, des Primes et Allocations de guerre. Pourtant dans ces premières années qui suivent le conflit rien n'est fait ni pour trouver une date commémorative, ni pour structurer un protocole particulier à suivre pour célébrer les morts.

Ces décisions émanent directement des anciens combattants. Ils font pression dès 1919 pour obtenir le 11 novembre comme le jour de commémoration. L'État finit par accepter cette date par la loi du 24 octobre 1922. Dès lors se met en place un cérémonial qui perdure jusqu'à nos jours. Réunis autour du monument aux morts, les anciens combattants, les endeuillés et la société communale répètent chaque année le même rituel. Discours, minute de silence, lecture de la liste des noms présents sur le monument et souvent la Marseillaise rythment cette journée. Ainsi se structure l'organisation de la commémoration. Pourtant si la forme reste quasiment inchangée, la teneur des propos tenus autour de ces édifices évolue en fonction des époques et donc du contexte.

$18 \mathrm{Au}$ lendemain de la Grande Guerre, les cérémonies commémoratives s'apparentent avant tout à des manifestations funéraires comme l'a expliqué Antoine Prost ${ }^{15}$. Il ne faut pas oublier qu'elles insistent également sur un aspect patriotique, en cherchant à glorifier les morts, ainsi que sur une dimension pacifiste. Si les anciens combattants cherchent à maintenir des cérémonies apolitiques, certaines idéologies transparaissent pourtant dans les discours des autorités locales. Ce fut déjà le cas lors de certaines inaugurations des constructions, à l'image de celle de la commune de Coublevie. Lors de cette cérémonie, les joutes politiques furent parfois plus identifiables que le recueillement, comme en témoigne le Petit Voironnais du 12 août 1923 :

$\mathrm{Au}$ dessert, [...] courte allocution de M. Mistral, député [de la SFIO], ajoutant à son souvenir aux morts une note politique qui n'a dû satisfaire que ses coreligionnaires 
de parti ; [...] et magnifique harangue de M. le sénateur Rivet [du parti radical], vibrante d'éloquence patriotique, [...] M. Rivet, glorifie, en termes élevés, la foi patriotique de la Nation, dont le cœur bat si parfaitement avec le cœur de ceux qui ont la lourde direction de ses destinées, et faisant une courte allusion aux agitations intérieures soulevées par le Communisme, il souligne de son mépris l'insignifiance de cette exaltation en rendant un magnifique hommage à l'Amour pour la Patrie ${ }^{16}$.

Figure 5

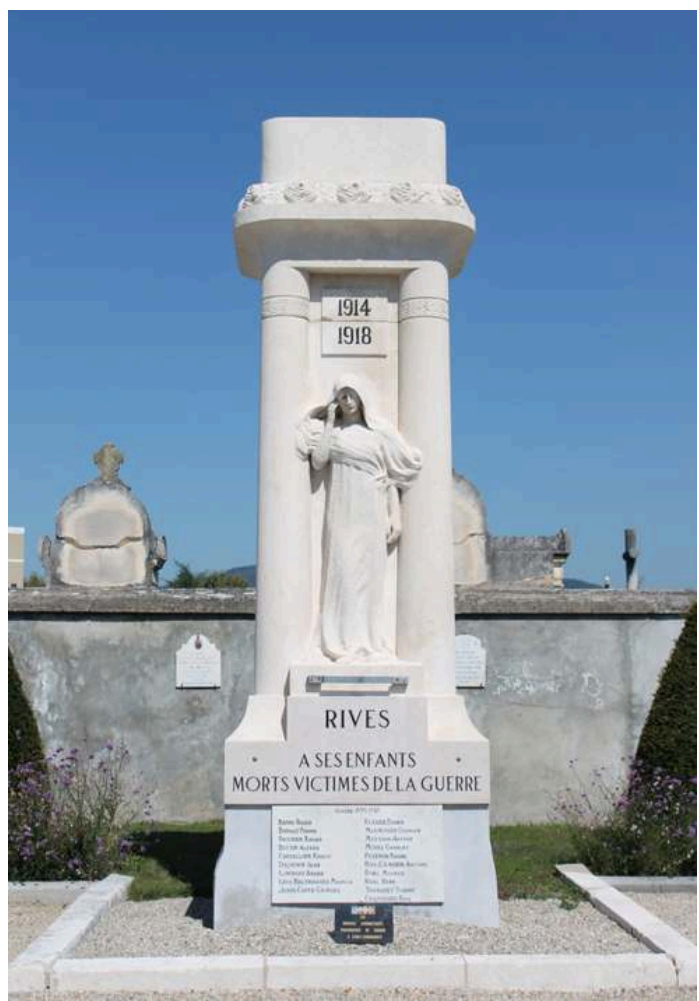

Rives (Isère), monument aux morts situé dans le cimetière, 2013.

Phot. Pignard, Jérémy. (c) Jérémy Pignard.

Ces divergences se retrouvent également avec le monument en lui-même. Dans la commune de Rives en Isère, deux monuments de la Grande Guerre sont présents. Le premier se situe dans le cimetière (fig. 5) ; initié par la municipalité de gauche au lendemain de la guerre, il insiste sur les sentiments pacifiste et funéraire. En désaccord avec ce courant de pensée, la majorité de droite accédant à la tête de la ville en 1919 fait édifier un second monument, plus patriotique, à proximité de l'église (fig. 6). Ainsi l'Union sacrée qui s'était déjà effritée à la fin de la guerre, continue-t-elle à se dissoudre au début des années 1920 . Se pose ici un paradoxe puisque si le monument aux morts rassemble des personnes aux opinions religieuses et politiques différentes, voire opposées, les discours tenus à ses abords témoignent au contraire de véritables prises de position. Ces caractéristiques politiques sont très importantes car les commémorations prennent des aspects qui peuvent varier en fonction de l'orientation politique de la commune. Certaines ont insisté sur la nécessité de la paix alors que d'autres ont entretenu un sentiment patriotique et parfois nationaliste. Cependant le sens politique n'est pas le seul à orienter la teneur des cérémonies commémoratives. 


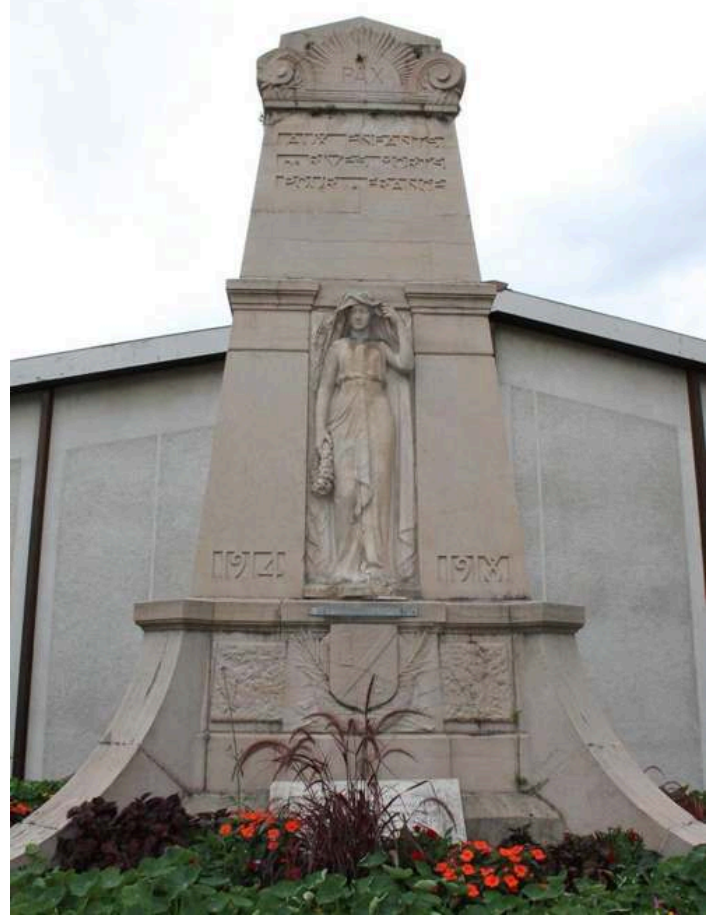

Rives (Isère), monument aux morts situé à proximité de l'église, 2013.

Phot. Pignard, Jérémy. ( ) Jérémy Pignard.

20 Le contexte joue un rôle prépondérant dans les commémorations. Le maire de la commune de Lux, dont il a déjà été question, insiste sur le contexte international lors de la cérémonie du 11 novembre 1933 :

Tout jusqu'à ce jour n'a été pour nous que désillusions, que concessions sans résultat positif ; c'est ainsi que, cédant à la pression exercée par Genève par certains de nos anciens alliés trop soucieux de leurs intérêts personnels qui viennent trop souvent s'opposer aux nôtres. Ce fut d'abord l'abandon de la Ruhr, [...] et enfin tout dernièrement le coup de poing sur la table du Führer sur la question du désarmement de la France, alors que l'Allemagne, elle, continue ouvertement ses armements pour être prête à nous envahir dès qu'elle trouvera le moment propice ${ }^{17}$.

21 Le monument aux morts est une tribune d'où il est possible de commenter l'actualité et d'en faire une analyse orientée. Ainsi le souvenir des morts n'est pas le seul leitmotiv de l'édifice. Ce dernier fait office de catalyseur d'idées, de vecteur de messages qui dépendent de la période mais également des personnes qui les portent. De ce fait chaque édifice peut être interprété de différentes manières et son utilisation peut évoluer dans le temps. Ces aspects sont d'autant plus importants lorsqu'il intègre de nouvelles mémoires.

22 Avec le second conflit mondial se pose la question de savoir comment célébrer ces nouveaux «morts pour la France». Si certaines communes font le choix d'ériger de nouveaux édifices spécifiques au contexte, la grande majorité décide d'ajouter ces noms à la liste de 1914-1918. Ce pragmatisme s'explique avant tout par un souci financier ; dans une période d'après-guerre marquée par les restrictions et les nécessités de reconstruction, le souci de l'hommage aux morts est secondaire. De plus cette pratique 
empirique est rendue possible par le faible nombre de noms à ajouter, le plus souvent une ou deux personnes. Dès lors le monument aux morts regroupe différentes mémoires et cela s'accentue avec les guerres de décolonisation dans lesquelles est impliquée la France.

Les monuments qui célèbrent les soldats de la guerre d'Indochine «morts pour la France » de manière spécifique sont rares (bien qu'il soit à noter qu'un mémorial national existe à Fréjus) et leurs noms sont souvent ajoutés à la longue liste des morts des deux guerres mondiales. Le fait qu'il n'y ait que très peu de constructions faisant référence aux soldats de ce conflit s'explique par plusieurs hypothèses. En premier lieu ces victimes sont des militaires de carrière. Or jusque-là seuls les "soldats de circonstance $»^{18}$ étaient commémorés sur les monuments aux morts. Ensuite, ce conflit est une défaite pour l'armée française et les monuments aux morts commémoraient généralement des victoires (même si celle de 1939-1945 est particulière). Dès lors se pose la question de savoir si l'on peut honorer de la même façon des vainqueurs et des vaincus.

Figure 7

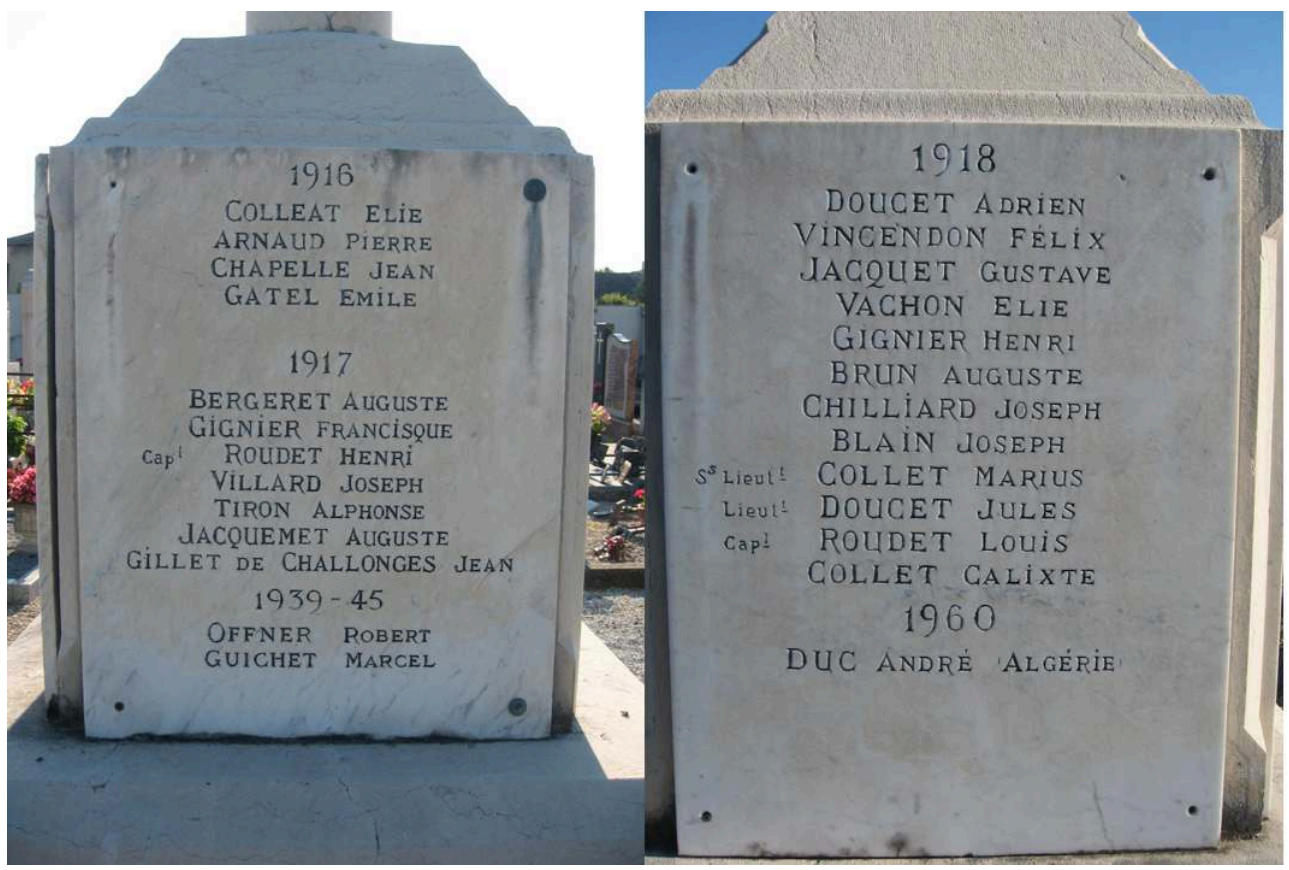

Brezins (Isère), détail des inscriptions du monument aux morts du cimetière, 2013.

Phot. Pignard, Jérémy. (c) Jérémy Pignard.

Le même problème est soulevé avec la guerre d'Algérie qui inscrit également des "soldats de circonstance" puisque c'est le contingent qui est envoyé pour le "maintien de l'ordre ». L'épigraphie des monuments aux morts explicite bien les spécificités de chaque conflit en précisant à chaque fois le conflit au cours duquel est morte la victime. Elle témoigne également d'un «syncrétisme mémoriel », puisque l'édifice concentre un mélange d'influences provenant des survivants des différents conflits et de leurs mémoires. La multiplication des dates commémoratives (8 mai, 19 mars) ne fait qu'accentuer ce phénomène. Si les cérémonies sont quasiment identiques, elles témoignent à chaque fois de la spécificité du conflit commémoré à travers les discours. Le monument aux morts du cimetière de Brézins en Isère (fig. 7) 
insiste sur ce pluralisme des mémoires; un second monument au sein de cette commune offrant même le souvenir d'un autre conflit qu'est la guerre de 1870 (fig. 8). De fait, ces édifices concentrent les différentes mémoires des victimes des guerres. Il demeure par conséquent au centre du patrimoine de la commune car il reste un objet mémoriel utilisé que l'inscription des victimes militaires françaises au cours des conflits actuels veut renforcer.

\section{Figure 8}

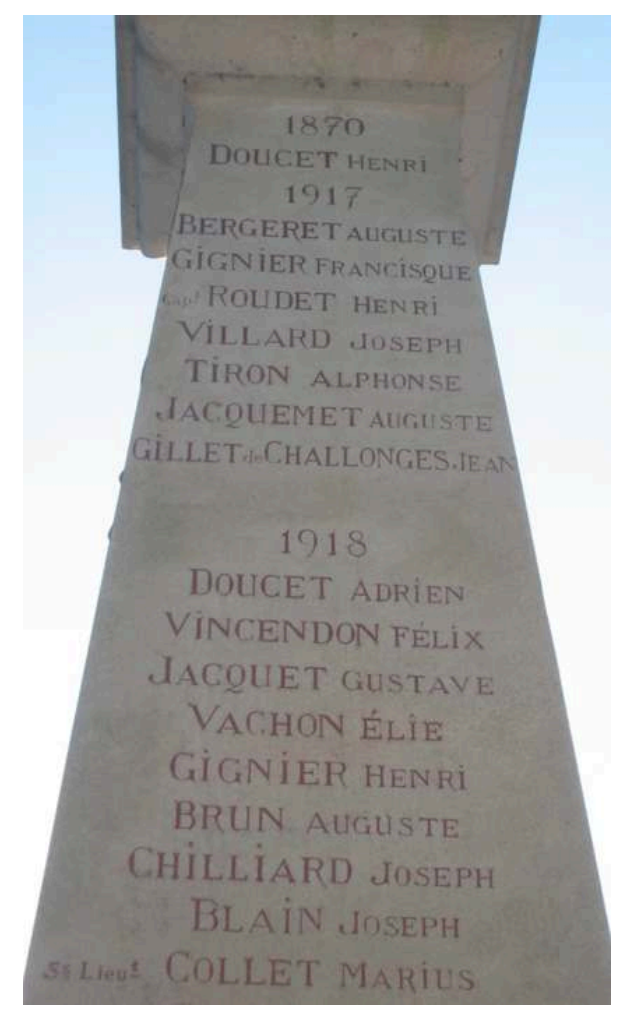

Brezins (Isère), détail des inscriptions du monument aux morts situé sur une place de la commune, 2013.

Phot. Pignard, Jérémy. (c) Jérémy Pignard.

Lors des conflits actuels qui émaillent le monde moderne, la politique de la mémoire change. Tout d'abord ces guerres n'ont plus lieu sur le territoire national, ce qui nuance l'idée du soldat combattant mort pour son pays (ici la mention «mort au nom de la France » ne prendrait-elle pas plus de signification que «mort pour la France»?) Ensuite les combattants servant dans l'armée française sont à présent tous des soldats de carrière, ayant fait le choix de servir leur patrie. Dès lors se produit une nouvelle donne mémorielle puisque ces hommes et ces femmes qui meurent lors d'opérations en territoire extérieur ne sont plus ces «soldats de circonstance » rencontrés lors des deux conflits mondiaux et de la guerre d'Algérie.

Pourtant, il paraît évident aux yeux des politiques comme de la société que ces combattants doivent recevoir eux aussi un hommage national en reconnaissance de leur sacrifice ; après tout, ils sont morts au nom des valeurs défendues par la France. Mais se pose rapidement la question de la forme que doit revêtir cet hommage. Peut-on réunir ces nouveaux noms sur les édifices communaux sans risquer de créer un amalgame entre les différentes mémoires ? Comment commémorer par le même objet 
un paysan mobilisé et mort à Verdun en 1916 et un soldat de carrière, ayant fait le choix de s'engager dans l'armée ${ }^{19}$, mort au cours d'une mission au Mali ? Si aujourd'hui nous avons les capacités de distinguer les différents types de personnes présentes sur ces édifices, en sera-t-il de même dans cinquante ans? Autant de questions qui rendent délicat le choix de l'hommage à adopter.

La première commune de France à avoir franchi le pas est celle de Saint-Aupre en Isère. Elle a inauguré le 11 novembre 2011 une nouvelle inscription sur le monument communal (fig. 9) rendant hommage à l'un de ses ressortissants, Clément Chamarier, mort en opération en Afghanistan en février 2011. Inscrire ce nom au côté des héros / victimes de la Grande Guerre, du second conflit mondial et des guerres en Afrique du Nord place ce soldat dans le "Panthéon communal». Toutefois, comme le dit le maire de la commune ${ }^{20}$, il a été important de préciser que ce soldat est "mort en Afghanistan " pour bien éviter tout amalgame avec les autres noms sur le monument. Si tous les morts de la commune tombés au cours des différentes guerres sont réunis sur ces édifices, il est en effet nécessaire de spécifier le conflit au cours duquel ces personnes sont tombées afin d'éviter toute forme de confusion. Si l'initiative de cette commune fut inédite, le procédé a été rapidement institutionnalisé par la loi du 28 février $2012^{21}$ rendant obligatoire l'inscription sur les monuments aux morts des personnes ayant reçu la mention « morts pour la France ».

Figure 9

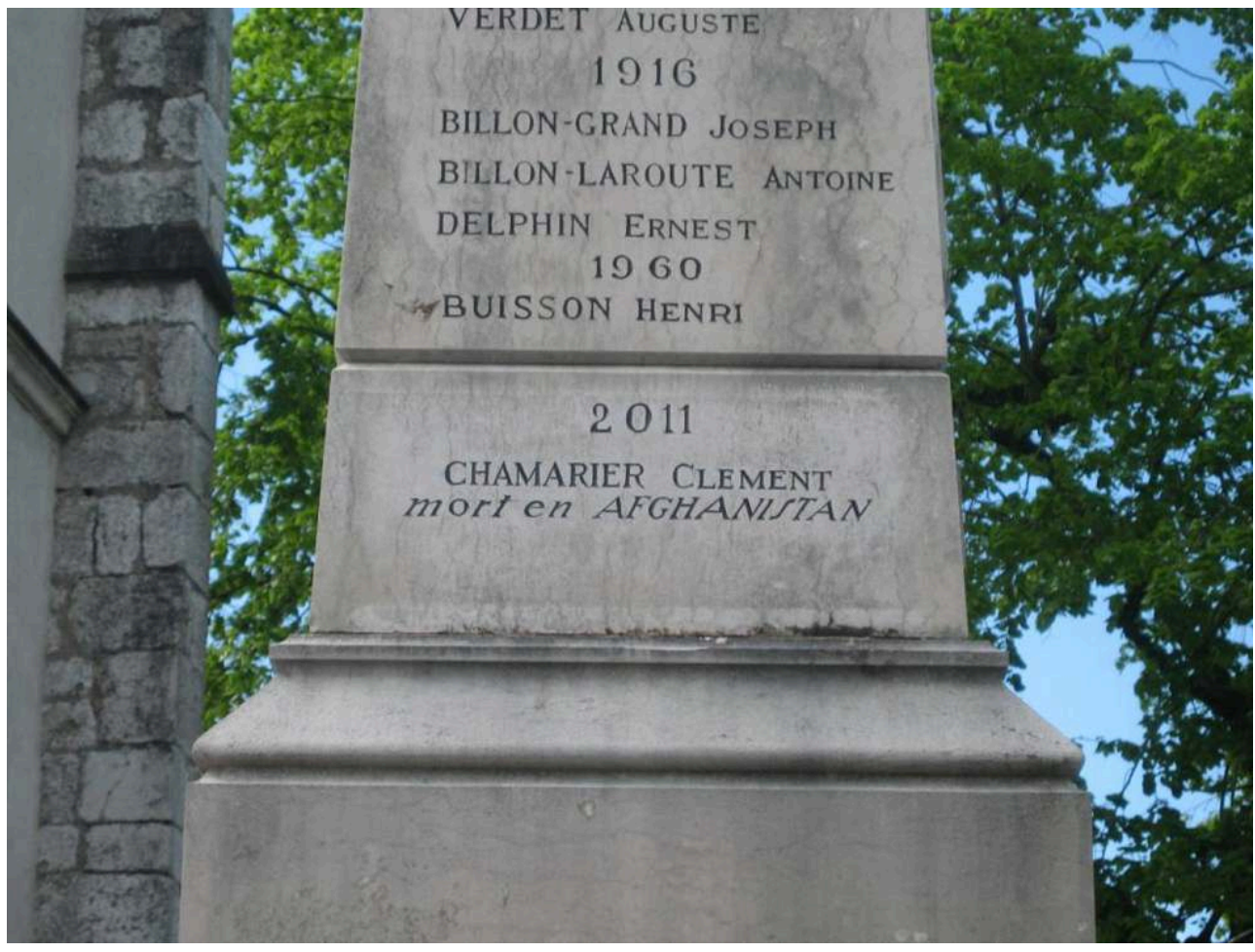

Saint-Aupre (Isère), détail du monument aux morts, 2012.

Phot. Pignard, Jérémy. (c) Jérémy Pignard.

Ainsi cette nouvelle utilisation du monument aux morts peut tendre à renforcer sa place au cœur des pratiques mémorielles actuelles. Au-delà du symbole le plaçant comme lieu d'un culte laïc et commémoratif d'événements passés, il devient le centre d'un hommage contemporain. Le message qu'il porte, même s'il fut élaboré il y a près 
de cent ans, reprend force avec l'actualité. Cela peut expliquer le retour des personnes autour des monuments lors des dates commémoratives. Pour les jeunes générations, l'édifice devient plus un objet d'apprentissage civique qu'une source historique. Cette construction conserve sa fonction: être à la fois l'un des pôles de la mémoire communale et une partie de la mémoire nationale. Toutefois ces constructions sont également les dépositaires d'une mémoire "à tiroirs" dans le sens où ils rendent hommage à des victimes de plusieurs conflits. En effet les architectes et responsables communaux ont bien pris garde de ne pas créer un amalgame entre les guerres par l'utilisation de symboles ou d'inscriptions réaffirmant la spécificité de chaque conflit. Pour autant, cet artifice sera-t-il suffisant pour permettre aux générations futures de comprendre les différences entre les nombreux noms et éviter ainsi toutes les formes d'amalgame?

\section{NOTES}

1. - PROST, Antoine. "Les monuments aux morts. Culte républicain? Culte civique ? Culte patriotique?». Dans NORA, Pierre (dir.). Les Lieux de mémoire, tome 1 - La République. Paris: Gallimard, 1984, p. 195 à 225.

2. - CABANES, Bruno. La Victoire endeuillée. La sortie de guerre des soldats français (1918-1920). Paris : Seuil, collection L'Univers Historique, 2004.

3. - Le Petit Voironnais, dimanche $1^{\mathrm{er}}$ juin 1919 - « Aux morts glorieux de la Guerre ». Consulté aux archives départementales de l'Isère (ci-après ADI), PER799/17.

4. - AUdOUIN-ROUZEAU, Stéphane, BECKER, Annette. La Grande Guerre 1914-1918. Paris: Découvertes Gallimard, 1998.

5. - KOSELLECK, Reinhart. L'expérience de l'Histoire. Paris : Seuil/Gallimard, 1997.

6. - La République de l'Isère, dimanche 16 mars 1919 - Moirans, "À la mémoire des héros morts pour la France ». ADI, PER741/50.

7. - La République de l'Isère, lundi 13 septembre 1919 - Miribel-les-Échelles, « En l'honneur de nos morts ». ADI, PER741/51.

8. - PROST, Antoine. «Les monuments aux morts. Culte républicain? Culte civique ? Culte patriotique?». Dans NORA, Pierre (dir.). Les Lieux de mémoire, tome 1 - La République. Paris: Gallimard, 1984.

9. - La République de l'Isère, lundi 29 septembre 1919 - Bévenais, « Le monument aux morts ». ADI, PER741/51.

10. - Articles L488 à L493 bis du Code des pensions militaires d'invalidité et des victimes de guerre.

11. - Archives privées, Discours prononcé par le maire de Lux (Côte-d'Or) le 11 novembre 1926 au cimetière de la commune.

12. - Le Petit Voironnais, dimanche $1^{\mathrm{er}}$ avril $1923-1^{\mathrm{e}}$ page, "À propos du monument aux morts ". ADI, PER799/19.

13. - Le Petit Voironnais, dimanche 2 septembre $1923-1^{\mathrm{e}}$ page, «L'inauguration du Monument aux morts de Saint-Aupre - Une bonne leçon aux communistes ». ADI, PER799/19.

14. - BARCELLINI, Serge. "La politique de la mémoire patriotique ». Historiens et Géographes, octobre $1986, \mathrm{n}^{\circ} 311$, p. 75 à 83. 
15. - PROST, Antoine. «Les monuments aux morts. Culte républicain? Culte civique? Culte patriotique?». Dans NORA, Pierre (dir.). Les Lieux de mémoire, tome 1 - La République. Paris, Gallimard, 1984, p 195 à 225.

16. - Le Petit Voironnais, dimanche 12 août $1923-1^{\mathrm{e}}$ page, « Inauguration du Monument aux morts de Coublevie ». ADI, PER799/19.

17. - Archives privées, Discours prononcé par le maire de Lux (Côte-d'Or) le 11 novembre 1933.

18. - Ici cette expression est faite pour bien différencier les soldats de métier de ces hommes et de ces femmes qui ont combattu pour la France uniquement à cause du contexte ; ainsi nous parlons des mobilisés en 1914-1918 et 1939-1940, des résistants, des appelés du contingent en Algérie... Parmi ces "soldats de circonstance " sont présents des militaires de carrière mais l'ampleur du conflit auquel ils ont participé les ont fait incorporer la mémoire globale.

19. - Ce qui signifie qu'il reçoit un salaire pour cette fonction et que sa mission fait partie de cette profession même.

20. - Entretien réalisé le 5 mai 2012, en mairie de Saint-Aupre, de M. G. Bouffard Roupé, maire de Saint-Aupre par J. Pignard.

21. - Loi $n^{\circ} 2012-273$ du 28 février 2012 fixant au 11 novembre la commémoration de tous les morts pour la France - Article 2 rendant obligatoire l'inscription sur les monuments aux morts des personnes ayant reçu la mention « morts pour la France ».

\section{ABSTRACTS}

As has been suggested by the work of various historians working on this topic, war memorials are places with a multitude of functions. Today they serve as the leading medium for the commemoration of the conflicts of the past. But they are also places where private and collective grieving can be given expression. A memorial building is an element of a municipal heritage, a parish heritage or even sometimes a private heritage. If all these places are considered together, they are clearly an integral part of the national heritage. Although they do not always elicit unanimous approval, they remain the main scene for the commemorative ceremonies in which the nation expresses its values. This is clearly suggested by the addition to these existing war memorials of the names of the professional soldiers who have been killed in recent overseas operations.

Les monuments aux morts sont des espaces plurifonctionnels, comme l'ont bien montré les travaux de nombreux historiens spécialisés sur le sujet. Aujourd'hui ils sont un support majeur de la mémoire des conflits passés. Mais ils sont aussi un espace d'expression du deuil privé et collectif. Un édifice seul est un élément du patrimoine communal, paroissial ou même parfois privé. Si l'on envisage l'ensemble de ces édifices, on s'inscrit alors dans un patrimoine national. Si ces constructions n'ont pas toujours fait l'unanimité, elles demeurent le cadre majeur des cérémonies commémoratives où se refonde la Nation: l'insertion de soldats maintenant professionnels morts sur les théâtres d'opérations extérieures de nos jours en est une preuve patente. 
INDEX

Keywords: war memorials, Isère, memory, war

Mots-clés: monument aux morts, Isère, commémoration, mémoire, guerre, syncrétisme mémoriel

\section{AUTHOR}

\section{JÉRÉMY PIGNARD}

Professeur d'histoire-géographie dans le secondaire, doctorant UMR LARHRA - Grenoble jf.pignard@wanadoo.fr 